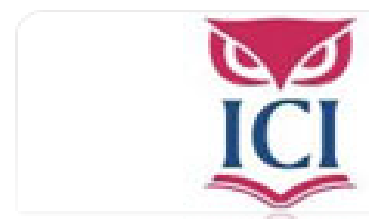

IUS. Revista del Instituto de Ciencias Jurídicas de Puebla A.C.

ISSN: 1870-2147

revista.ius@hotmail.com

Instituto de Ciencias Jurídicas de Puebla A. C.

México

Fernández Olazábal, Pedro

Transexualidad, homosexualidad y familia (reflexiones teóricas y resultados de trabajo desde una visión de la psicología)

IUS. Revista del Instituto de Ciencias Jurídicas de Puebla A.C., núm. 20, 2007, pp. 26-41

Instituto de Ciencias Jurídicas de Puebla A. C.

Puebla, México 


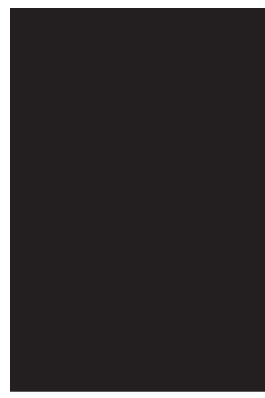

\section{TRANSEXUALIDAD, HOMOSEXUALIDAD Y FAMILIA (REFLEXIONES TEÓRICAS Y RESULTADOS DE TRABAJO DESDE UNA VISIÓN DE LA PSICOLOGÍA)}

Pedro Fernández Olazábal*

I. Los trastornos SEXUALES (REFLEXIONES té́ricAs) II. LA FAMILIA COMO GRUPO SOCIAL Y SU ROL EN LA FORMACIÓN DE LA PERSONALIDAD Y LA SEXUALIDAD III. Caracterización psicológica de la familia del transexual Y EL HOMOSEXUAL (RESULTADOS DE TRABAJO)

iv. Conclusiones

\section{RESUMEN}

El autor presenta reflexiones teóricas en torno a la conceptualización de los trastornos sexuales, su diferenciación con variantes de la sexualidad humana y la visión crítica desde las posiciones de la psicología de la personalidad. Esta visión personológica, facilita las valoraciones acerca del papel de la familia en el desarrollo de la personalidad y específicamente de la esfera sexual, en la cual se concibe la misma como contexto del desarrollo, independientemente de su estructura y tipo. Muestra como resultado una caracterización psicológica de la dinámica familiar con respecto a variantes de la sexualidad y los trastornos sexuales.

\section{ABSTRACT}

It presents theoretical reflections around the conceptualization of the sexual dysfunctions, their differentiation with variants of the human sexuality and the vision criticizes from the positions of the psychology of the personality. This vision of the personality, facilitates the valuations about the paper of the family in the development of the same one and specifically of the sexual sphere, in which the same one is conceived as context of the development, independently of its structure and type. It shows a psychological characterization of the family dynamics as a result with regard to variants of the sexuality and the sexual dysfunctions.

'Doctor en ciencias psicológicas, Universidad de Camagüey, Cuba. Profesor adjunto de la Escuela Libre de Psicología de Puebla. 


\section{Los trastornos SeXuales (RefleXiones teóricas)}

La Clasificación Internacional de las Enfermedades en su décima revisión, conocida como CIE 10, clasifica en sus categorías principales los trastornos referidos a la vida sexual del ser humano. Considera para su descripción como dimensiones fundamentales de la sexualidad la identidad sexual (CIE10, Epígrafe F64), la inclinación sexual (CIE-10, Epígrafe F65), el desarrollo sexual y los trastornos, problemas o situaciones patológicas asociadas a la orientación sexual (CIE-10, Epígrafe F66). Cada una de estas dimensiones se refiere a aspectos esenciales de la vida sexual, aunque en la citada clasificación no se especifican conceptualmente las mismas.

Así, por ejemplo, la identidad sexual, devenida como resultante de la identidad de género, y ésta a su vez de los niveles subjetivos de la identidad queda sólo al nivel de sus trastornos o distorsiones, sin valorar su dimensión general, que resulta vital para la reflexión que en este artículo se referirá y para la comprensión de la sexualidad humana en su dimensión psicológica y sociocultural.

Por este motivo, se valorará cada dimensión desde su concepción general para después especificar los trastornos.

Identidad sexual: Es el resultado de la valoración de la correspondencia entre el sexo biológico y la construcción psicosocial de género asociada, como estereotipo a ese sexo biológico. Es la interiorización, la estructuración y consolidación al nivel personológico individual, es decir al nivel de la organización psicológico-subjetiva individual del conjunto de procesos psicológicos que garantizan la conceptualización del yo como varón o hembra, como hombre o mujer. Este contenido interiorizado, como cualidad psicológica individual, matiza todo el sistema de autopercepción, autoconceptualización, autovaloración y autorreflexión del sujeto en correspondencia con los grupos de hembras o varones. Es la percepción del sistema de relaciones y de pertenencias y referencias con esos grupos. Según E. Barberá (1998), "es el proceso por el que cada cual se sabe perteneciente a un grupo de asignación sexual y excluido del otro". Como podrá apreciarse, en la identidad sexual hay un alto compromiso para el yo, para la autoconcepción de sí mismo. Se centra en la subjetividad, en el sistema de autovaloraciones del sujeto, rebasando el contexto concreto de las relaciones sexuales, y el placer sexual va más allá del acto concreto referido al coito, los juegos sexuales o la excitación sexual. 
De esta manera los llamados trastornos de la identidad sexual, implican una inconformidad con el sexo biológico y la construcción de género asignada. El sujeto vivencia diferencias que provocan malestar, incompatibilidad sexo-género, dificultades en la integración a grupos sexualmente asignados e imposibilidad para incorporar los patrones de conducta genérica esperados según su sexo biológico. La incompatibilidad con el yo llega al nivel del trastorno, de distorsión del autoconcepto y de ruptura con la identidad esperada, así como enfrentamiento en lo social para asumir la identidad deseada. En resumen la identidad sexual trastornada es una forma de no ser lo que se debería ser, y a la vez, no ser lo que se ansía ser.

La búsqueda del placer queda "diluida, expandida o quizás enmascarada en la asunción de la identidad y las formas de vida del sexo deseado y no concretamente en la relación sexual. En este acápite el CIE 10 reconoce los siguientes trastornos:

- Transexualismo (F64.0): Consiste en el deseo de vivir y ser aceptado como un miembro del sexo opuesto, que suele acompañarse por sentimientos de malestar o desacuerdo con el sexo anatómico propio y de deseos de someterse a tratamiento quirúrgico u hormonal para hacer que el propio cuerpo concuerde lo más posible con el sexo preferido. Es un trastorno estable, cuyas pautas para el diagnóstico conciben que la identidad transexual debe haber estado presente, constantemente y de forma estable, por lo menos durante dos años, y no ser un síntoma de otro trastorno mental, como esquizofrenia, o acompañar a cualquier anomalía intersexual, genética o de los cromosomas sexuales.

- Trasvestismo no fetichista (F64.1): Consiste en llevar ropas del sexo opuesto durante una parte de la propia existencia a fin de disfrutar de la experiencia transitoria de pertenecer al sexo opuesto, pero sin ningún deseo de llevar a cabo un cambio de sexo permanente y menos aún de ser sometido a una intervención quirúrgica para ello. Debe ser distinguido del trasvestismo fetichista, en el que hay una excitación sexual acompañando a estas experiencias de cambio de vestido.

- Trastorno de la identidad sexual en la infancia (F64.2): Se trata de trastornos que suelen manifestarse, por primera vez, durante la primera infancia (siempre antes de la pubertad), caracterizado por un malestar intenso y persistente debido al sexo propio, junto 
al deseo (o insistencia) de pertenecer al sexo opuesto. Hay una preocupación constante con el vestido o las actividades del sexo opuesto, o un rechazo hacia el propio sexo. Se cree que estos trastornos son relativamente raros y no deben confundirse con la falta de conformidad en el papel sexual socialmente aceptado, que es mucho más frecuente. El diagnóstico del trastorno de la identidad sexual requiere una profunda alteración en el sentimiento normal de masculinidad o feminidad. No es suficiente la simple masculinización de los hábitos en las chicas o el afeminamiento de los chicos. Es un deseo general y persistente de ser, o insistencia de que se es del sexo opuesto al propio, junto a un intenso rechazo del comportamiento, atributos y atuendos del mismo.

Los trastornos difieren en sus manifestaciones clínicas, comportamentales y psicológicas, pero mantienen como denominador común el conflicto con el yo, con el sí mismo y con el autoconcepto, variables psicológicas que dan al traste con la integración del sujeto a grupos, a la vida social, a la vida afectiva, a las relaciones interpersonales y por supuesto a la familia, grupo especial que se caracteriza por ser el punto diana, el origen y el condicionante principal de muchos de los trastornos o situaciones del desarrollo de la personalidad.

La inclinación sexual: Se refiere a la excitación sexual, a la búsqueda del placer, a la satisfacción imperiosa de necesidades sexuales relacionadas con objetos, partes del cuerpo, situaciones o fenómenos considerados como objetos sexuales propiamente dichos, como provocadores o facilitadores de la excitación sexual. Difiere de la identidad por estar pegada y determinada por la satisfacción de necesidades concretas, necesidades de placer y dependencia de los objetos o situaciones generadores de la excitación sexual.

El análisis psicológico del problema, desde la perspectiva personológica, choca ineludiblemente y desde una primera aproximación, con el término inclinación sexual. Este término, desde cualquier perspectiva resulta ambiguo, poco definido y poco esclarecedor del problema que pretende abordar. A criterio del autor, al referir esa dimensión de la sexualidad, se trata de necesidades sexuales, y no de inclinación a algo; se trata de deseos irreprimibles, de impulsos incontrolables con respecto a objetos, personas 
o situaciones no concebidas en el ámbito sociocultural como portadoras de connotación sexual o erótica.

La necesidad, como carencia, sólo se incentiva y adquiere sentido, cuando se encuentra con el objeto que potencialmente la satisface. Es este encuentro necesidad-objeto, quien facilita que este último devenga en motivo de la actividad, que a su vez se orientará a la satisfacción de la necesidad. Por tanto, más que inclinación se trata de necesidad y motivo, de la necesidad a satisfacer y del motivo que induce la actuación, a la búsqueda del objeto deseado, obviando relaciones sociales, familiares y grupales, así como los aspectos de la cultura pautados socialmente.

No se refiere a un compromiso del yo, o de la identidad, sino que refleja los aspectos específicos de la búsqueda del placer, al cual puede vivirse de manera obsesiva, esporádica, poco frecuente o sistemática. De cualquier manera pone en peligro la estabilidad y el equilibrio psicológico del sujeto, al menos durante un periodo, la estabilidad y el equilibrio de la familia y llega hasta la peligrosidad social. La diferencia además se centra en la forma de vivenciar el trastorno y en la configuración personológica del sujeto: en los casos de compromiso a la identidad la vivencia tiende al sufrimiento, al vivir neurótico, a la idea fija e irreductible de ser o querer ser del otro sexo, con aislamiento, autorrechazo y evasión de la realidad. En el caso de los trastornos de las necesidades o inclinación sexual, se trata de vivir en la búsqueda del placer, en la búsqueda del objeto que satisface la necesidad o facilita la excitación.

En este caso el CIE-10, en su epígrafe F65, contempla como trastornos a la generalidad de las parafilias, y se destacan entre ellas, el fetichismo, (F65.0), el trasvestismo fetichista (F65.1), el exhibicionismo (F65.2), la escoptofilia (F65.3), la paidofilia (F65.4), el sadomasoquismo (F65.5), los trastornos múltiples de la inclinación sexual (F65.6), otros trastornos de la inclinación sexual (F65.8) y los no especificados (F65.9).

Cada uno de estos trastornos difiere en el objeto o la situación deseada y necesaria para la excitación y el placer sexual, pero tienen en común la necesidad, la vivencia de dependencia y la búsqueda de ese placer; además el contacto con el objeto es la fuente fundamental y en ocasiones única de placer y excitación sexual. Puede acompañarse de vivencias circunstanciales de culpa, pero no compromete la identidad, ni el sujeto vivencia el trastorno de forma abierta ante la sociedad.

Estos trastornos tienen una repercusión a largo o corto plazo, pero de gran intensidad en la familia y los grupos de pertenencia del sujeto, so- 
bre todo cuando implican daños o molestias a terceros, y por supuesto se acompañan de un sistema o conjunto de situaciones estigmatizantes para la familia a nivel de la sociedad.

El desarrollo y la orientación sexual: El desarrollo sexual del ser humano es pluridimensional. Tiene que ver fundamentalmente con una dimensión biológica, psicológica o psicosexual y social. Su punto más elevado o crítico se da en la adolescencia, periodo en el que los cambios puberales, el desarrollo y la maduración de las estructuras anatómico-sexuales y los procesos fisiológicos se dan de forma vertiginosa, trayendo consigo una fuerte repercusión en la estructuración de la personalidad en formación del adolescente, de su estructuración psicológica y la definición de muchos de sus contenidos. La adolescencia es un momento decisivo en la conformación de la identidad sexual, en el reconocimiento y el surgimiento de necesidades sexuales y en el desarrollo psicosexual del sujeto. En este periodo se dan grandes pasos para el reconocimiento y la consolidación de la orientación sexual futura. La orientación sexual no es patológica en sí, es un reconocimiento de la singularidad y la individualidad de cada cual con respecto a su sexualidad. Se refiere a las vías por las cuales transcurre la vida sexual del ser humano, a su sistema de preferencias, expectativas, satisfacciones y sentido personal de la relación amorosa-sexual con personas del sexo opuesto o del mismo sexo.

En su conformación implica un complejo proceso de interiorizaciónexteriorización, reflexiones y autorreflexiones, valoraciones del mundo, de los demás y de sí mismo, confrontaciones con grupos de pertenencia, la familia como un grupo de este tipo por excelencia, vivencias de compromisos afectivos y morales, valoración y enfrentamiento con estereotipos sociales, tradiciones, costumbres y prejuicios; es el choque con la intersubjetividad que le antecede, con la cultura y las exigencias sociales, así como con los estereotipos de género. La orientación sexual es contenido esencial y necesario de la identidad sexual, pero no la supera como concepto en su grado de alcance y amplitud.

Se reconocen como formas de la orientación sexual: la heterosexualidad, la homosexualidad, la bisexualidad y otras, entre las que se incluyen las puberales. Si bien ellas no constituyen trastornos en sí mismas, sí se pueden acompañar de varios de ellos, entre los que se destacan los referidos a la egodistonía, como elemento distorsionador. Así se señala en la clasificación la orientación sexual egodistónica (F66.0), concebida como la inconformidad o inseguridad adquirida con la orientación sexual. Se con- 
cibe, además, el trastorno de la relación sexual (F66.2), como consecuencia de dificultades o distorsiones de la identidad o la orientación, y los trastornos de la maduración sexual (F66.0), en los cuales, más que un fenómeno madurativo lo afectado es el reconocimiento de una naciente orientación sexual, o la estabilidad de la supuestamente conformada con anterioridad. Pone en peligro la conciencia de identidad, en el caso del adolescente, y la estabilidad de la orientación, en el caso del adulto. No se trata, a criterio del autor, de una maduración dañada, acelerada o tardía, sino de un sistema personológico, subjetivo individual, que se dinamiza, se transforma y genera nuevas necesidades y motivos, provocando una situación de crisis, en ocasiones antagónica entre lo nuevo a reconocer y los habituales patrones de comportamiento aceptados en lo personal y lo social.

Son trastornos o situaciones profundamente vivenciales para el sujeto, y con gran repercusión en la familia y los grupos de pertenencia, implican cambios sustanciales de formas de vivir, nuevas formas de reconocimiento del sí mismo, autoaceptación y autovaloración, así como un fuerte compromiso para el sí mismo, el yo y los sistemas valorativos de la sociedad.

De forma general la descripción de los trastornos sexuales referidos en la clasificación tiene en cuenta fundamentalmente aspectos de índole psicológica, tales como identidad psicológica y de género, conducta, necesidades, motivaciones, orientación sexual, sentidos personales, el yo, el sí mismo y otras. Por tanto se impone la valoración de los contextos de desarrollo psicológico, de la situación social del desarrollo y de los agentes encargados directamente de la mediación social y la socialización como proceso formador de la personalidad.

En este sentido se hace necesaria la valoración de los aspectos teóricos de los trastornos sexuales, lo cual es condición indispensable para su análisis objetivo y la influencia en ese proceso. Se deben tener en cuenta los factores reguladores externos, como la familia y los grupos y sus potencialidades como futuros contenidos autorreguladores.

Sólo el esclarecimiento de estos aspectos del nivel teórico permitirá la valoración del papel de la familia, como integrante y conductora de cualquier situación social del desarrollo psicológico y de la formación de la personalidad, permitiendo alejarse de las consabidas, y ya casi manidas, recomendaciones para el tratamiento o manejo familiar y en la familia de cualquier trastorno, situación o crisis de la vida de un sujeto, sobre todo en el contexto de las acciones con objetivos educativos, de salud o supuestamente desarrolladores. 


\section{LA FAMILIA COMO GRUPO SOCIAL Y SU ROL EN LA FORMACIÓN DE LA PERSONALIDAD Y LA SEXUALIDAD}

La familia, como grupo, cumple una función esencial de mediación entre el individuo y la sociedad, es decir de mediadora y agente socializador por excelencia. Es el núcleo primario del hombre, donde surgen y se desarrollan las primeras vivencias afectivas, la primera percepción de identidad y de diferenciación del yo, con respecto a otros seres humanos y al medio circundante. Es en la familia donde se aprueban, estimulan, refuerzan o desaprueban las primeras instancias comportamentales, las normas de conducta social y las primeras relaciones interpersonales, y es en ella donde todas estas dimensiones del funcionamiento psicológico y social del ser humano van adquiriendo sentido personal. Es en la familia donde se pautan las primeras nociones de género e identidad sexual, donde el niño va incorporando, según la interiorización de las valoraciones de los adultos afectivamente significativos para él, los sistemas de expectativas de género, los patrones de autoaceptación y de adecuación a normas sociales. Esta interacción es vital para la conformación de estructuras de personalidad, contenidos psicológicos, reconocimiento de necesidades y motivos de conducta relacionados con una "sexualidad sana".

La concepción de la sexualidad, como cualidad moral, como instancia moralizante o desmoralizante, es en gran medida conformada y legalizada por la familia. De esta manera se desarrollan y afianzan en el sujeto opiniones, valoraciones, normas, prejuicios y estereotipos, que acompañarán al deseo y las motivaciones sexuales durante toda la vida o gran parte de ella. Es así precisamente como se producen las instancias psicosociales, inicialmente como reguladores externos y posteriormente como instancias autorreguladoras del funcionamiento personológico y sexual del sujeto.

La familia, como grupo social, ha sufrido y sufre cambios esenciales. Esta realidad, por una parte legaliza su carácter dinámico y por otra la convierte en unidad de análisis y punto de reflexión para el estudio y la comprensión de los problemas del hombre. En ella se dan confrontaciones entre valores, entre "valores emergentes" y "valores tradicionales" (Arés, M. P., 2002). Así, lo emergente, lo nuevo, resultado de una época, es lo crítico, lo no aceptado, lo que falta por probar y que da al traste con la tradición, generadora de seguridad. No obstante la familia se ha modificado y se modifica, tanto en su dimensión estructural, como en la psicológica, planteándole a las ciencias encargadas de su estudio y a los profesionales en la práctica la tarea de 
deconstruir su concepto tradicional. Según Arés (2002), "tras esa deconstrucción el concepto de familia queda para algunos autores definido como la unión de personas que comparten un proyecto vital de existencia en común, que se quiere duradero, en el que se generan fuertes sentimientos de pertenencia a dicho grupo, existe un compromiso personal entre sus miembros y se establecen intensas relaciones de intimidad, reciprocidad y dependencia".

El concepto enfatiza en la unión de personas, la comunidad de objetivos y proyectos, los sentimientos de pertenencia al grupo y las intensas y profundas relaciones afectivas entre los miembros. Es precisamente a esto a lo que está llamada la familia, a la formación de la personalidad, la comprensión y el respeto a la individualidad.

Estos valores intrínsecos del grupo familiar, que emergen como prioritarios y necesarios para una época nueva, en detrimento de valores tradicionales centrados en lo formal externo de la familia y su imagen social, determinan y a la vez son expresión de los cambios por los que atraviesa la familia como institución social. Ilustra estos cambios la concepción de que para ser considerada familia, no sea condición indispensable el matrimonio legal, el tener hijos, o la unión rígida y para toda la vida de la pareja original. De hecho la familia decide si tiene hijos o no, si la pareja continúa o si se reestructura o reensambla la familia, la mujer decide si cría los hijos en compañía de un hombre o no, incluso las personas deciden el sexo preferido de su pareja.

Varios países han aprobado el matrimonio entre personas del mismo sexo, y se discute la adopción de niños y niñas por parejas formadas por personas del mismo sexo.

La llamada familia homoparental, formada por una pareja del mismo sexo, ya sean dos mujeres o dos hombres, reclama cada vez más su lugar en la sociedad y el espacio para el cumplimiento de sus funciones como familia. El diario español El País, en número de junio de 2006, refleja estadísticas de adopción y convivencia de niños por familias homoparentales, de un 1\%, en países como Holanda y Reino Unido, así como en Estados Unidos de América. Se estima que en España la cifra asciende a los 80,000 menores que viven bajo la tutela de este tipo de familia. Además estas parejas, en busca de su reconocimiento social, han fundado e integran una asociación, ${ }^{1}$ cuyo objetivo es promover la crianza de los hijos, su educación y socializar sus derechos a la familia.

${ }^{1}$ La Asociación de Familias Gay y Lesbianas con aHijos e Hijas (Galehi, www.galehi.org), diario El País, lunes 26 de junio de 2006. 
Resultados científicos (Del Mar, M, 2006) ${ }^{2}$ corroboran que la diversidad familiar es un hecho, cuyos modelos están constituidos no sólo por las familias homoparentales, sino también por las monoparentales y las reconstituidas. En el caso de la homoparental se realizan estudios longitudinales acerca de su capacidad y potencialidad para la educación de los hijos, frente a los modelos que niegan esta realidad y defienden la capacidad educativa y el cumplimiento de funciones sólo para el modelo tradicional de familia.

La referida psicóloga y la Academia Americana de Pediatría, después de años de trabajo en Estados Unidos, Canadá, Reino Unido, Suecia y Bélgica, publicó en un número especial de su revista del año 2000, que existe equivalencia entre el desarrollo de los niños criados por familias homoparentales y los criados por familias heterosexuales. En su estudio consideró los siguientes indicadores:

- Presencia de perfiles personales sanos.

- Capacidad educativa adecuada.

- Dinámica familiar saludable o funcional.

- Apoyo social suficiente.

El análisis de estos indicadores en las familias homoparentales estudiadas arrojó resultados positivos a su favor, por lo que se consideró que tenían las posibilidades para ser consideradas como "contexto adecuado para el desarrollo". Los resultados no pretenden elevar la familia homoparental por encima de la heterosexual tradicional, sino demostrar que la presencia de trastornos, incapacidad educativa y la disfuncionalidad familiar pueden presentarse en un tipo de familia u otro, pues no dependen directamente de la orientación sexual. Del Mar (2006), en un estudio comparativo de ambos grupos de familias detectó que las homoparentales son más igualitarias en la distribución de roles genéricos y en las relaciones entre sus miembros. Señala, además, que los padres y madres de estas familias llegan a la maternidad o paternidad de una manera siempre más reflexiva y programada que las heterosexuales, pues en ellos se excluye el papel de la casualidad y el fallo de métodos anticonceptivos. Se detectó, como debilidad, la carencia de referentes y el rechazo social que aún persiste en determinados sectores poblacionales o contextos socioculturales.

${ }^{2}$ María del Mar González, especialista en psicología evolutiva y de la educación, Universidad de Sevilla, España. 
En entrevista realizada a Jesús Santos, presidente de Galehi, refiere que la asociación pretende lograr un espacio en la sociedad, defendiendo su modelo de familia frente al absolutismo del modelo tradicional. A la vez considera que la legalización del matrimonio ha repercutido positivamente en la familia homoparental y en la adopción de niños, pero todavía no de la manera que se esperaba.

Lo esencial es el cumplimiento de las funciones que como familia, independientemente de su estructura, ésta debe cumplir. Entre esas funciones se citan: la función biológica, la económica y la cultural-espiritual, las cuales en sistema traen como resultado el cumplimiento de su función esencial o suprafunción: la educación para la vida.

La función educativa de la familia, su rol en la formación de la personalidad y la sexualidad, emerge como un argumento insoslayable, con la aparición en el contexto científico de la psicología y la pedagogía con enfoque histórico cultural, donde comienzan a quedar rezagadas las tendencias que absolutizaban el individualismo del sujeto o el papel absoluto del medio en la formación de la personalidad.

Así, la sexualidad es el resultado de un proceso de socialización-individualización, en el cual lo social deviene individual, mediado por los agentes encargados de la transmisión: la familia, la escuela, y los otros (las demás personas).

Si bien no se aspira a tener o formar la familia perfecta, al menos sí se aspira a la más querida y valorada. Por otra parte tampoco existen las recetas para la familia, ni son absolutamente ciertos los listados de conductas o situaciones esencialmente negativas y dañinas. Para cada cual su familia tiene aspectos positivos y otros no tanto, pero a cada cual su familia le legó algo que lo acompañará por el resto de su vida. Esto es muy válido para la sexualidad, para la capacidad de amar, para la maternidad y la paternidad y sobre todo para la vida en común con otras personas.

En el análisis de la familia y su rol en el desarrollo sexual del sujeto cobran vida conceptos tales como: límites y espacios vitales, respeto a la individualidad, estereotipos y prejuicios, expectativas de la familia, idealización y desidealización del otro y de los hijos, proyección de los padres en los hijos, autorrealización personal, concepción del mundo, concepción de la pareja, concepción de normalidad y anormalidad y patrones morales.

La familia como agente promotor de la situación social del desarrollo psicológico, debe tener en cuenta para el caso específico del desarrollo sexual de los niños y adolescentes, los siguientes aspectos: 
- La valencia del sistema de relaciones afectivas y de comunicación que el niño establece con las figuras del sexo correspondiente a su sexo biológico, es decir, como la figura del padre o la madre, genera relaciones potencialmente adecuadas con las representaciones de género y que éstas sean aceptadas y valoradas por el niño.

- La asociación posible entre figuras potencialmente promotoras del desarrollo sexual y su valencia positiva, negativa o contradictoria en el proceso de formación de los sentidos personales.

- La transmisión de los roles y comportamientos de género y su asociación con vivencias placenteras o displacenteras para el niño.

- La educación sexual como contexto que respeta límites, espacios vitales y posibilidades para la expresión de la individualidad y el crecimiento personal.

- El tratamiento y la comunicación a los temas de la sexualidad sin prejuicios, ni dogmas, pero a la vez sin otorgarle demasiada relevancia, cinismo o concebirla como necesidad y obligación de una etapa del desarrollo.

- Las manifestaciones de afectividad y cariño sin distinción de sexo, y sin que el rol de género sea una limitante para esa manifestación. Es el caso de las relaciones entre padre e hijos varones.

- Considerar el amor no posesivo, ni el hijo como propiedad en la cual se realizan los padres, sino como ente individual con derechos e identidad propia y diferente.

\section{Caracterización psicológica de la familia del transexual Y EL HOMOSEXUAL (RESULTADOS DE TRABAJO)}

Este epígrafe se ha dedicado a la caracterización de la familia del transexual (concebida la transexualidad como trastorno) y la del homosexual (concebida la homosexualidad como variante de la orientación sexual). La selección se debe a la dispar frecuencia de aparición de ambos fenómenos, presentándose en la realidad mayor frecuencia para la homosexualidad. Además debido a las falsas concepciones que a nivel popular, e incluso en comunidades científicas ajenas a la psicología y la psiquiatría, aún persisten. Presenta resultados del trabajo, durante varios años, como orientador 
de familias en el plano de la sexualidad y la crianza de los hijos. Estos resultados se han agrupado esencialmente en una síntesis de las actitudes, posiciones o situaciones de la dinámica psicológica y relacional de la familia, con respecto a un miembro de ella, por lo general un hijo o hija homosexual.

De las situaciones que con mayor frecuencia aparecen en las familias que demandan este tipo de orientación se señalan:

- Carencia o distorsión de los conocimientos acerca del desarrollo sexual de los niños, y de su participación como familia en este desarrollo.

- Depósito en el hijo de las expectativas de la familia.

- Idealización de los hijos, según estereotipos, normas y valores legalizados por esa familia.

- Carencia de conocimientos acerca de la diferenciación entre los trastornos sexuales, su origen y su concepción como trastorno.

- Predominio de los estigmas sociales y prejuicios morales al analizar los trastornos sexuales.

- Confusión e identificación entre orientación sexual y trastornos sexuales.

- Consideración de la orientación sexual heterosexual como sana y la homosexual como trastorno.

- Confusión entre transexualidad y homosexualidad, siendo lo más relevante para la familia, la atracción por el mismo sexo.

- No distinción entre preferencia por el rol genérico y atracción sexual.

- Presencia de estigmas morales que acompañan a la homosexualidad y los homosexuales, y análisis prejuiciado del caso.

- Predominio de una supuesta aceptación del fenómeno en su dimensión abstracta, pero rechazo cuando éste se presenta en la propia familia.

- En el caso del transexual, se produce una falta total de comprensión del trastorno, sus características y su funcionamiento psicológico, el cual se concibe como capricho o problema moral. 
- La familia del transexual se centra más en los problemas del rol de género y los estigmas sociales que acompañan a estos cambios que en la propia atracción sexual del sujeto.

- Específicamente el transexual, más que el homosexual, está propenso a la segregación de la familia, quien no admite su forma de vida y evaden o atenúan la vergüenza excluyéndolo de su dinámica.

- Presencia de sentimientos culpabilizantes y búsqueda de la supuesta responsabilidad de algún miembro de la familia, como promotor o generador de la homosexualidad.

- En muchas familias una vez lograda la supuesta aceptación del homosexual, ésta es muy superficial, pues se canaliza a través de sentimientos de conmiseración hacia el "pobre enfermo".

- Es frecuente la aceptación resignada hacia el homosexual, la cual se impone por el cumplimiento del rol de madre o padre, "quienes nunca abandonarían a sus hijos".

- El homosexual se somete a un reclamo sordo de agradecimiento y chantaje emocional, debido a la aceptación de la familia, y está llamado a reconocer y agradecer la supuesta aceptación.

- Conciencia en el homosexual de "ser una carga bochornosa para la familia", quien además es muy bondadosa al admitirlo.

- Aceptación parcial, demostrada a través del silencio y la omisión de los problemas y vivencias del homosexual en el seno de la familia. Ésta se comporta como grupo de pertenencia a medias, al cual sólo puede pertenecer el homosexual, cuando muestra "su mejor lado de la vida" y por supuesto omite el otro.

- El homosexual tiende a buscar y construir grupos y espacios de pertenencia ajenos a la familia como contextos de autenticidad y escape al rechazo.

- La familia descarga su supuesta responsabilidad en la orientación sexual del hijo o la hija en las amistades y las relaciones negativas que ha establecido.

- Concepción aparente de la homosexualidad como orientación sexual normal, pero atribuida a supuestos traumas de la infancia, 
lo que denota un sentido personal contradictorio en la familia, al no considerar lo incoherente de la posición. La pregunta obligada sería: ¿cómo puede ser normal lo que surgió por la vía traumática?

- Supuesta aceptación al hijo homosexual, pero rechazo manifiesto o encubierto a su pareja.

- Presencia de sentimientos ambivalentes de aceptación-rechazo en la familia, y aceptación de algún miembro frente al rechazo de otros.

- Tendencia al ocultamiento del "problema".

- La valoración de la orientación sexual matiza todo el sistema de valoraciones de la familia hacia el homosexual, opacando o atenuando sus logros profesionales, personales o de cualquier otra esfera de la vida.

- La familia del homosexual tiende con frecuencia al pacto con éste: "vive como quieras, pero al menos no lo demuestres".

- Algunos miembros de la familia, básicamente el padre y la madre, vivencian profundamente una lucha de motivos, entre el cariño por el hijo o la hija homosexual y la imagen social.

- Pobre o ausente concepción acerca de la diferencia y la diversidad entre las personas. Relacionado con esto muestran, también, una valoración de la diferencia permeada del sentido comparativo de inferioridad-superioridad.

\section{iv. Conclusiones}

- La especificidad conceptual en las reflexiones teóricas acerca del desarrollo sexual, los trastornos sexuales y la sexualidad en general es de vital importancia para una adecuada comprensión del problema. Tal es el caso de la identidad sexual, la orientación sexual, las necesidades y motivaciones sexuales y los trastornos sexuales.

- La familia es el principal grupo promotor de contextos desarrolladores y de la situación social para la formación y desarrollo de la personalidad. De forma similar lo es para la 
sexualidad y el desarrollo sexual, como uno de los componentes estructurales y de contenido con alto potencial autorregulador en la vida y el funcionamiento psicológico autónomo del sujeto.

- La posición de la familia frente a la orientación sexual de los hijos está determinada y/o condicionada por contenidos reguladores externos, lucha de motivos afectivos y morales, carencia de conocimientos sobre el tema, y contradicción entre valores emergentes y valores tradicionales de la familia.

- La familia que demanda orientación psicológica, para estas situaciones, presenta características específicas de su dinámica y funcionamiento familiar, generadas por el estigma social que aún acompaña a la orientación sexual.

\section{REFERENCIAS}

Arés, M. P., Psicología de la familia. Una aproximación a su estudio, Editorial Félix Varela, La Habana, 2002.

Barberá, E., Psicología del género, Ariel, Barcelona, 1998.

E. de, Benito, "Las familias gay salen del armario. Nace una asociación para dar visibilidad y apoyo a las parejas del mismo sexo y sus hijos", diario El Pais, Madrid, lunes 26 de junio del 2006.

Fernández, O. P. y Pardillo P. J., "Psicodiagnóstico de Rorschach y estudios sobre género", en El Rorschach: Una visión integradora, Editorial Ácana. Colección Ciencia y Técnica, Camagüey, 2004.

Fernández 0. P., "Personalidad, familia y salud", conferencia curso de posgrado, Psicologia de la personalidad, Universidad de Camagüey, 2006.

Fernández, R. L., Pensando en la personalidad, tomos i y II, Editorial Félix Varela, La Habana, 2005.

Leontiev, A. N., Actividad, conciencia y personalidad, Editorial Pueblo y Educación, La Habana, 1982.

Martínez B. I. y Bonilla C. A., Sistema sexo/género, identidades y construcción de la subjetividad, Universitat de Valencia, Valencia, 2000.

oms, Trastornos mentales y del comportamiento, décima revisión de la Clasificación Internacional de Enfermedades, Descripciones clínicas y pautas para el diagnóstico, CIE 10, Meditor, España, 1992.

Prats. J., "Miles de personas viven en hogares con padres homosexuales", diario El País, Madrid, lunes 26 de junio del 2006.

Volver al Índice >> 